\title{
Pinging reveals active, not silent, working
}

\section{memories}

\author{
Joao Barbosa ${ }^{1,2}$, Diego Lozano-Soldevilla ${ }^{1,3,4}$ \& Albert Compte ${ }^{1 *}$ \\ ${ }^{1}$ Institut d'Investigacions Biomèdiques August Pi i Sunyer (IDIBAPS), C. Rosselló 149-153, 08036 Barcelona, Spain \\ ${ }^{2}$ Laboratoire de Neurosciences Cognitives et Computationnelles, Département d'Études Cognitives, École Normale \\ Supérieure, INSERM U960, PSL University, Paris, France \\ ${ }^{3}$ Centro de Investigación Biomédica en Red de Enfermedades Raras (CIBERER), Av. Monforte de Lemos 3-5, 28029 \\ Madrid, Spain \\ ${ }^{4}$ Laboratory for Clinical Neuroscience, Centre for Biomedical Technology, Universidad Politécnica de Madrid, Campus \\ de Montegancedo, 28223 Pozuelo de Alarcón, Spain \\ *Corresponding author: acompte@clinic.cat
}

Short Title: Pinging reveals active, not silent, working memories

\section{Abstract}

Persistently active neurons during mnemonic periods have been regarded as the mechanism underlying working memory maintenance. Alternatively, neuronal networks could instead store memories in fast synaptic changes, thus avoiding the biological cost of maintaining an active code through persistent neuronal firing. Such 'activity-silent' codes have been proposed for specific conditions in which memories are maintained in a non-prioritized state, as for unattended but still relevant short-term memories. A hallmark of this 'activity-silent' code is that these memories can be reactivated from silent, synaptic traces. Evidence for 'activity-silent' working memory storage has come from human electroencephalography, in particular from the emergence of decodability (EEG reactivations) induced by visual impulses (termed pinging) during otherwise "silent" periods. Here, we re-analyze EEG data from such pinging studies. We find that the originally reported absence of memory decoding reflects weak statistical power, as decoding is possible based on more powered analyses or reanalysis using alpha power instead of raw voltage. This reveals that visual pinging EEG 'reactivations' occur in the presence of an electrically active, not silent, code for unattended memories in these data. This crucial change in the evidence provided by this dataset prompts a reinterpretation of the mechanisms of EEG reactivations. We provide two possible explanations backed by computational models, and we discuss the relationship with TMS-induced EEG reactivations. 


\section{Introduction}

A hallmark of the activity-silent working memory framework [1] is that memories stored silently in synaptic traces through short-term synaptic plasticity can be reactivated through non-specific stimuli [1-5]. Evidence supporting activity-silent working memory has recently emerged from human electroencephalography (EEG) $[6,7]$, in particular from EEG reactivations of unattended memories induced by visual impulses [7] - the so-called visual pinging. Despite their relevance for upcoming memory-guided behavior, currently unattended memories could not be robustly decoded from raw EEG voltage traces [6,7] (Fig. 1A, red). In view of this, unattended memories resemble memories rendered behaviorally irrelevant by a contextual cue (discarded, Fig. 1B, dashed lines), but they differ from attended memories with similar upcoming behavioral requirements, which are represented in sustained, active codes [6-8] (Fig. 1A,B solid lines). This observation has been key in interpreting EEG reactivations in pinging studies as evidence for activity-silent storage (see for example recent reviews [8-14], or refs. [2-4,15] for explicit simulations of this interpretation of the data). Intriguingly, pinging-induced increase in EEG decodability occurred exclusively for items that remained relevant for future, memory-guided behavior, suggesting that only unattended but still relevant items were kept in activity-silent traces. The mechanisms for such selective switching of activity-silent memory are unclear, as in existing computational models of activity-silent storage $[1,2,4,5,16,17]$ short-term plasticity changes are induced by neuronal activity, regardless of its behavioral relevance. Here, we reanalyzed EEG recordings of these influential pinging studies $[7,18]$. We found that unattended memories, previously shown to be inaccessible from scalp EEG voltages despite remaining behaviorally relevant [7], could in fact be decoded from raw EEG voltage and robustly from alpha power signals. This reanalysis demonstrates that the original claim of a silent representation of unattended memories is not supported by the data, which instead show an active code and thus calls for a reinterpretation of pinging-induced increases in EEG decodability. Finally, we argue that the increase in stimulus decodability following an unspecific stimulus, seen in human [6,7,18-20] and monkey electrophysiological experiments [21,22], can be explained by network models with or without short-term plasticity based on ongoing active, not silent, neural representations. 


\section{Results}

\section{Attended and unattended working memories are robustly decoded from alpha power}

We realized that an earlier study had reported that (attended) spatial memories were decoded more reliably from EEG total alpha power than from evoked activity [23]. Thus, we analyzed alpha-power information content for attended, unattended and no-longer relevant orientation memories in the publicly available dataset of the original publication by Wolff et al. [7]. We found that a sustained alpha power code tracks the orientation of the items that remain relevant for future behavior, whether attended or unattended (Fig. 1C,D, solid lines, S1 Fig.). This shows that working memory contents are maintained in an electrically active neural code, even for items outside of the current attentional focus [24]. However, while attended memories were decodable both in alpha power and voltage traces, unattended memories were only robustly detected in alpha power. While this could reflect qualitative differences in what these two neural signals represent [25-27], we will explore here the parsimonious possibility that this stems from differential sensitivity of these two measures to the same underlying neural activity. Indeed, EEG voltage is known to lose decodability shortly following a reference baselining due to slow electrical drifts [28], while oscillations could be more robust to these baseline drifts. In this view, attended items would be represented by strong neural signals (represented both in voltage and in alpha power) while unattended items would be kept in analogous but weaker neural signals (picked only by alpha power). These neural dynamics could result from competitive interactions between prioritized and unprioritized memory items, in line with competing attractors in networks without activity-silent mechanisms [20,24,29].

\section{Lack of statistical power suggests spurious evidence for silent representations of unattended memories}

In line with the hypothesis of an active but weaker representation of the unattended items, recent studies $[30,31]$ show that lack of decodability for unattended working memories can be overcome by increasing statistical power (e.g. sample size). We wondered if a similar strategy could improve decodability of unattended orientations 
from raw voltages in this dataset. We addressed this tentatively in the current datasets with two complementary approaches. First, we attempted to increase the statistical power by 1) smoothing the voltage traces with a 32-ms kernel prior to decoding (instead of smoothing instantaneous decoding accuracies as done in the original study [7]; Methods), 2) averaging decoding accuracies over an interval of $200 \mathrm{~ms}$ before the pinging impulse, and 3) pooling trials from all sessions and subjects (Methods). After these extra preprocessing steps, we still failed to decode discarded or unattended memories from raw voltage (both $\mathrm{p}>0.16$, two-sided t-test; $t=1.38$ and $t=0.03$, respectively). We then tested the influence of sample size on decoding estimates in each condition, by varying the number of sessions and trials included in the analyses. This showed a striking difference between unattended and discarded memories: while increasing the sample size in the unattended condition resulted in a monotonic increase of $t$-value, it did not for discarded memories (see diagonal in Fig. 2). This result suggests that increasing the number of sessions would lead to decodability of unattended memories, but not of discarded memories. This motivated our second approach, in which we reasoned that the low signal-to-noise ratio in this cohort could be due to specific sessions with overall low decodability. Sessions with low decodability could reflect technical issues during that particular session (e.g. EEG sensor placement) or specific subject characteristics, such as skull thickness or hair density. We thus divided our full dataset using cross-validation in high and low decoding sessions, based on the average decoding accuracy during the early delay ('split period' in Fig. 3, Methods). We found that unattended memories could be robustly decoded during the whole delay and in particular immediately before pinging from high-decoding sessions, while discarded memories could not (Fig. 3). Finally, we show that sessions with high early-delay decoding ('split period' in Fig. 3) are also those that have 'reactivations' in voltage seen in the original publication (Fig. 3, inset). These analyses, together with previous studies showing robust decoding of unattended memory items $[22,30,32-37]$, suggest that also in this dataset unattended items are not stored in activity-silent traces. 
Two plausible explanations for the increase in decodability that do not require activity-silent mechanisms

If there is an active EEG code for both attended and unattended stimuli prior to the visual impulse, as our analyses suggest, then what is the interpretation of the observed increase in EEG decodability $[7,18]$ ? We reasoned that EEG reactivation events may emerge from either an increase in the signal about the stimulus (as assumed in the activity-silent interpretation) or through a reduction in the across-trial variability (S3C Fig.). In the data, we found that pinging reduces across-trial variability of EEG voltage (Fig. 4A), as expected for neural responses to sensory stimuli [38]. In addition, we found that trials with stronger EEG decodability showed lower across-trial variability than trials with weaker EEG decodability during pinging (Fig. 4B), demonstrating a link between trial-by-trial EEG variability and pinging-induced increase in EEG decodability. We argue that a reduction of variability with an otherwise intact active memory representation (Fig. 3) is a parsimonious interpretation of the visual pinging effect. Alternatively, there is another interpretation of pinging-induced increases in EEG decodability consistent with our findings. Recent modeling work has shown how the enhancement of active representations $[20,21]$ is expected when pinging recurrent neural networks with no need for activity-silent mechanisms [29]. An existing representation maintained in an attractor supported by recurrent and competitive interactions enhances its tuning when it is stimulated unspecifically (attractor-boost model, Fig. S2). Also this mechanism would be consistent with these data, as it shows reduced variability (Fig. $4 \mathrm{C}$, gray), concomitant with boosted attractor tuning (S2 Fig.). While both of these possible interpretations do not exclude an interplay of active representations with activity-silent mechanisms [17,39-41], they offer a parsimonious view that renders activity-silent working memory an inadequate framework to understand increases in decodability induced by non-specific stimuli [7,18,20,21]. To further support this, we sought to evaluate variability predictions from a computational model where reactivations occur because of factual memory reactivation from silent, synaptic traces. We tested an available biophysical network model for (continuous) activity-silent working memory [5], which is an extension of the canonical, but discrete model of activity-silent working memory [1] (Methods). In these simulations, a non-specific input induced reactivations in some trials, causing an increase of across-trial variability (Fig. 4C, black). This is because reactivations in such attractor 
networks are an all-or-none phenomenon, and great variability is expected when triggering them from weak, decaying activity-silent traces in noisy spiking networks. In sum, pinging reveals an underlying active memory, perhaps by reducing noise (Fig. 4A,B, S3C Fig.) in the presence of an active code (Fig. 1, 3) or by enhancing tuning in an active representation (S2 Fig.), but not by reactivating stimulus signals from silent traces.

\section{Differential mechanisms for pinging- and TMS-induced reactivations}

Increases of EEG decodability at the presentation of non-specific impulses have been shown to occur not only for visual impulses [7] but also for external perturbation with single-pulse TMS [6]. Despite apparent similarities, TMS perturbations impact working memory performance [5,6,42-44], while pinging does not $[7,45]$ (see also chapter 4 of [46]). This suggested that these approaches could be interacting with fundamentally different neural mechanisms [7,47]. Indeed, we found through the re-analysis of the data of ref. [6] that single-pulse TMS increases across-trial EEG variability (Fig. 4D), in contrast to the reduction observed upon visual pinging (Fig. 4A). Such increase in across-trial variability is in accordance with the activity-silent working memory model presented before (Fig. 4C, black), thus supporting the interpretation of TMS EEG reactivations as signals recovered from activity-silent traces $[5,6]$. However, a note of caution is in order: the difficulty in precisely locating the TMS coil in different trials may strongly contribute to increased EEG variability, which could mask the true effect of TMS on EEG variability.

\section{Discussion}

Through the reanalysis of existing datasets, we provide here evidence that working memory 'reactivations' by visual pinging, considered prime evidence for activity-silent working memory [2-4,8-15], occur in the presence of active (not silent) ongoing memory representations in the delay period. In addition, we verify that representations for unattended items are notably weaker than for attended items, consistent with biased competition between active memories [29]. Based on this substrate for working memory, visual pinging may increase EEG decodability through (1) ping-induced reduction in across-trial variability, and/or (2) ping-induced boosting of attractor tuning. We further compare visual pinging with TMS perturbations, and we find qualitative differences suggesting different underlying 
mechanisms. Based on the difference in behavioral impact of these two perturbation protocols (visual pinging does not affect working memory behavior, but TMS does), we speculate that visual pinging may increase EEG decodability via reduced across-trial variability or by transient boosting of active attractors, while TMS-induced reactivations would be supported by activity-silent mechanisms. Note that temporarily boosting an active attractor should not have a strong impact on behavior beyond the boosting period (unless additional long-lasting cellular mechanisms are engaged), while true reactivations from activity-silent stores should have a long lasting impact, as the silent trace is refreshed.

We show here that relevant memories are stored in active codes, and are thus decodable, while irrelevant memories could not be decoded, therefore potentially discarded. This interpretation sheds light on the intriguing ineffectiveness of pinging on discarded memories, while being effective in 'reactivating' unattended but still relevant ones [7]. The reactivation effectiveness of pinging appears now to depend on whether memories are maintained in active neuronal representations, decodable from EEG. In line with this, a recent study shows that pinging reveals diffusing dynamics [45], a hallmark of active memories [45,48], instead of decaying dynamics, as expected in the activity-silent framework $[1,45]$. Our data reconciles the influential study by Wolff et al. [7] with recent works showing unattended memories actively encoded in scalp EEG [32-34], in the activity of cortical association areas using large-sample fMRI analyses [30] or intracranial recordings in monkeys [22,49], and in neural activity in visual areas of rodents [37]. While there is extensive evidence for long-lasting cellular and synaptic mechanisms in cortical neurons ('silent' mechanisms, e.g. [50]) that must coexist [51] with, or even support [17,40,52-54], active representations such as those reported in this study, there is more scant evidence that working memory can be volitionally stored without spiking activity $[1,55]$.

To avoid confounds with active representations, we [5] and others [56] have found evidence for activity-silent traces from previously memorized but now irrelevant items, for which chance-decoding is expected in principle. There, too, it is hard to discard low-powered designs, so positive evidence must be sought. We have recently reported that, between consecutive trials - when the previous memory should be discarded, similarly to uncued memories in ref. [7]-, neurons fire more 
synchronously after having been engaged in active working memory storage [5], suggesting that discarded memories can leave involuntary silent traces. Importantly, selecting sessions based on good overall decodability (as in Fig. 3) did not reveal decodability of these across-trial discarded memories (Fig. 1 in [5]), supporting a true activity-silent substrate. We argue that the effect of induced reactivations must be validated during similar conditions in which memories are demonstrably discarded, presumably leaving an activity-silent trail. Current evidence shows across-trial behavioral effects of TMS, as is expected by reactivating previous, discarded memories [5,43], but not of visual pinging during inter-trial periods (chapter 4 of [46]). Also the evidence in Fig. 3 suggests that visual pinging cannot reactivate putative activity-silent traces following a discarded item.

Finally, our results suggest that voltage and alpha power encode similar working memory content. Previous work, however, shows that alpha and raw voltage play different roles in working memory [25-27], in particular that alpha power tracks spatial attention instead of the actual memory content. In principle, with the experimental design of Wolff et al. [7], subjects could re-code orientation as a spatial location, which would be tracked by alpha power and by sustained voltages [25]. Future work with other experimental designs including independent variation of orientation and attentional location [25] could clarify this point further. Regardless of whether EEG is tracking orientation or a location re-codification, we argue that in these data both signals are carrying analogous contents, with voltage being noisier.

In sum, our results add to previous literature showing robust decoding of unattended working memories from electrophysiological signals [22,30,32-37]. Our analyses reinforce the idea that interpreting null decoding as evidence for storage in silent traces is not straightforward, because null results might result from weak signals in insufficiently powered analyses [30,31].

\section{Methods}

\section{EEG experiments}

We analysed 2 available datasets of visual pinging [7,18]. For decoding and EEG variability analyses, we focused on both experiments from [7]. In experiment 1 $(n=30)$, subjects were cued for which item was going to be probed (cued item, here 
also called attended, or uncued item, here called discarded memory). In experiment $2(n=19)$, subjects had to alternate their attention between 2 items (their early/late, here attended/unattended memory item). Experiment 1 consisted of one session, while experiment 2 consisted of two sessions (separated by approximately 1-2 weeks) on the same set of subjects. For variability analyses, we also analysed the experiment of ref. [18] $(n=24)$. In this experiment, the subjects had to memorize 1 item, thus always within the focus of attention. Importantly, the item decodability from raw voltage never dropped to chance. Additionally, we also analyse the voltage variance of the experiment $2(n=6)$ of a TMS study [6]. We refer the reader to the original studies for extra details $[6,7,18]$. All these datasets were made available in a fully anonymized format and had been approved by the corresponding institutional review boards, as indicated in the original publications.

Data preprocessing. The data available online was epoched and baselined relative to the beginning of each epoch (S3 Fig.). To revert this baselining, we computed trial-by-trial voltage difference between consecutive epochs. We then added this voltage difference to the beginning of each baselined epoch, effectively reverting all baselining effects. Additionally, for the variability analyses (see below) we remove any signal drift caused by, for example, moving electrodes using the python function scipy.signal.detrend on each subject's variability. Finally, for the decoding analysis (see below), we also computed the alpha power. For this purpose, the data was Hilbert-transformed (using the FieldTrip function " $\mathrm{ft}$-freqanalysis.m") to extract frequencies in the alpha-band $(8-12 \mathrm{~Hz})$ and total power was calculated as the squared complex magnitude of the signal.

Decoding analyses. We used freely available code to perform these analyses, so we will only briefly describe the methodology here. For a detailed description of the decoding methods, please refer to the original study [7]. As in the original study, we decoded from all the 17 posterior channels (P7, P5, P3, P1, PO7, PO3 and O1 versus P8, P5, P6, P4, P2, PO8, PO4 and O2). Briefly, we collected the Mahalanobis distance between all possible pairwise combinations of the orientations and thus form a representational dissimilarity function. Finally, the decoding strength was calculated as the vector strength of this function. We decoded from raw voltage or alpha power with the exact same code. The decoding strengths (Fig. 1) were smoothed over time with a gaussian kernel ( $S D=10 \mathrm{~ms})$. 
Across-trial variability analyses. We computed variability as the variance (var) across trials of the raw voltage traces (trials $x$ sensors $x$ time). Before averaging variances across sensors, we detrended them using the function scipy.signal.detrend to account for any drift in the signal. Finally, we computed the percentage of variability change ( $\Delta v$ var) relative to the baseline period of $2 \mathrm{~s}$ before the pinging stimulus $(b): \Delta v a r=(v a r-b) / b * 100$. This referencing to the baseline ensures that changes in variability can be attributed solely to the pinging and not to other factors that are common to both pre and after pinging, such as varying stimulus orientations.

Fano-factor. To compute the variability drop in the simulated spiking activity we used the fano-factor [38], which is defined as the variance of spike counts in a given window (100 ms) divided by their mean. We then computed $\Delta \mathrm{FF}$, as the difference relative to the baseline period of $2 \mathrm{~s}$ before pinging stimulus.

\section{Phenomenological simulations of EEG trials}

To study how single-trial baseline correction impacts pinging-induced increases in EEG decodability we applied our decoders to synthetic EEG data generated by a model where spurious EEG reactivations are caused by a reduction in noise variability. First, we simulated two hypothetical delay maintenance EEG time series representing two independent experimental conditions (i.e. grating oriented $0{ }^{0} \mathrm{~V}$.s. $45^{\circ} ; n=200$ each condition) using the following Gamma function (f) as a single-trial waveform:

$$
f(t \mid a, b)=\frac{e^{a-1}}{[b(a-1)]^{a-1}} t^{a-1} e^{\frac{-t}{b}}
$$

For each trial and condition parameters $a$ and $b$ were drawn from a Gaussian distribution (condition $1, \mu_{\mathrm{a}}=2, \mu_{\mathrm{b}}=130 \mathrm{~s}$; condition $2, \mu_{\mathrm{a}}=3, \mu_{\mathrm{b}}=80 \mathrm{~s}$; all conditions with $\sigma_{\mathrm{a}}=0.2, \sigma_{\mathrm{b}}=0.5 \mathrm{~ms}$ ). Each waveform was then scaled by 0.5 and 0.25 , respectively and the time onset was set to time point $0.1 \mathrm{~s}$.

Finally, for each single-trial and condition, Gaussian noise was added $(\mu=0, \sigma=$ 0.75). During the impulse period (1.3 s $-1.5 \mathrm{~s})$, for each trial and experimental condition, we reduced $30 \%$ the variability of the noise $(\sigma=0.525)$. 
To use the same decoding method as the original publication, we generated a multi-channel time series. We created two sets of dipoles located around left (position [1.5 -8.6 1.5] cm, orientation [-1 - -1 -1]) and right (position [1.5 -8.6 1.5] cm, orientation [ $\left.\left[\begin{array}{lll}1 & -1 & -1\end{array}\right]\right)$ primary visual cortex and the simulated time courses were projected to the scalp via a forward model [57]. Fig. S3,C plots decoding of these signals upon different conditions of EEG voltage baselining. The Matlab code for these simulations is available on https://github.com/comptelab/reactivations.

\section{Activity-silent network model}

We used a previously proposed computational model [5] to simulate memory reactivations. The model consists of a network of interconnected 2048 excitatory and 512 inhibitory leaky integrate-and-fire neurons [58]. This network was organized according to a ring structure: excitatory and inhibitory neurons were spatially distributed on a ring so that nearby neurons encoded nearby spatial locations [59]. Excitatory connections were all-to-all and spatially tuned, so that nearby neurons with similar preferred directions had stronger than average connections, while distant neurons had weaker connections. All inhibitory connections were all-to-all and untuned. Network parameters were taken from [5]. Simulation of "activity-silent" mechanisms was done by simulating two presynaptic variables $\mathrm{x}$ and $\mathrm{u}$, as described in [5]. Reactivations were accomplished stimulating all excitatory neurons with a non-specific external stimulus [5].

\section{Attractor-boost network model}

For the attractor-boost model we used a bump attractor model similar to the activity-silent model described above, but without short-term plasticity. As in this other model, attractor boosting was achieved with a non-specific external stimulus to all excitatory neurons. The Brian [60] code for this model is available on https://github.com/comptelab/reactivations. 
Improving statistical power

Smoothing. To improve signal-to-noise ratio for our decoding analyses we smoothed the voltage traces using a gaussian kernel with $\sigma=32 \mathrm{~ms}$. This was in contrast with the original study that used instantaneous, non-smoothed voltages for decoding and smoothed the resulting decoding accuracies with $\sigma=16 \mathrm{~ms}$.

Pooling all trials, across different sessions. We also pooled all trials across sessions and subjects. Because all subjects and sessions consisted of a similar number of trials, we are not biasing our analyses towards a specific subject. Note that we only pooled trials across sessions for the analyses in Fig 2. We averaged across sessions for the other analyses.

Cross validated median-split. To simulate an increase of signal-to-noise ratio, we removed sessions with low decodability. Importantly, to avoid circularity in our analysis we cross validated this selection in the following way. For each session, we split the trials in two halves. With the first half, we sorted the sessions by their decoding accuracy during early delay $[0.2-0.4] \mathrm{s}$, and we selected high and low decoding sessions (median split). We then computed the average decoding accuracy along the trial for low and high-decoding sessions in the second half. We repeated this procedure 1000 times and then plotted the $90 \%$ C.I. of decoding accuracies for the high and low-decoding sessions. Performing the delay-split with and without cross validation did not change the results qualitatively (not shown). For the reactivation split (Fig 3, inset), we thus ran the analysis without cross-validation. 

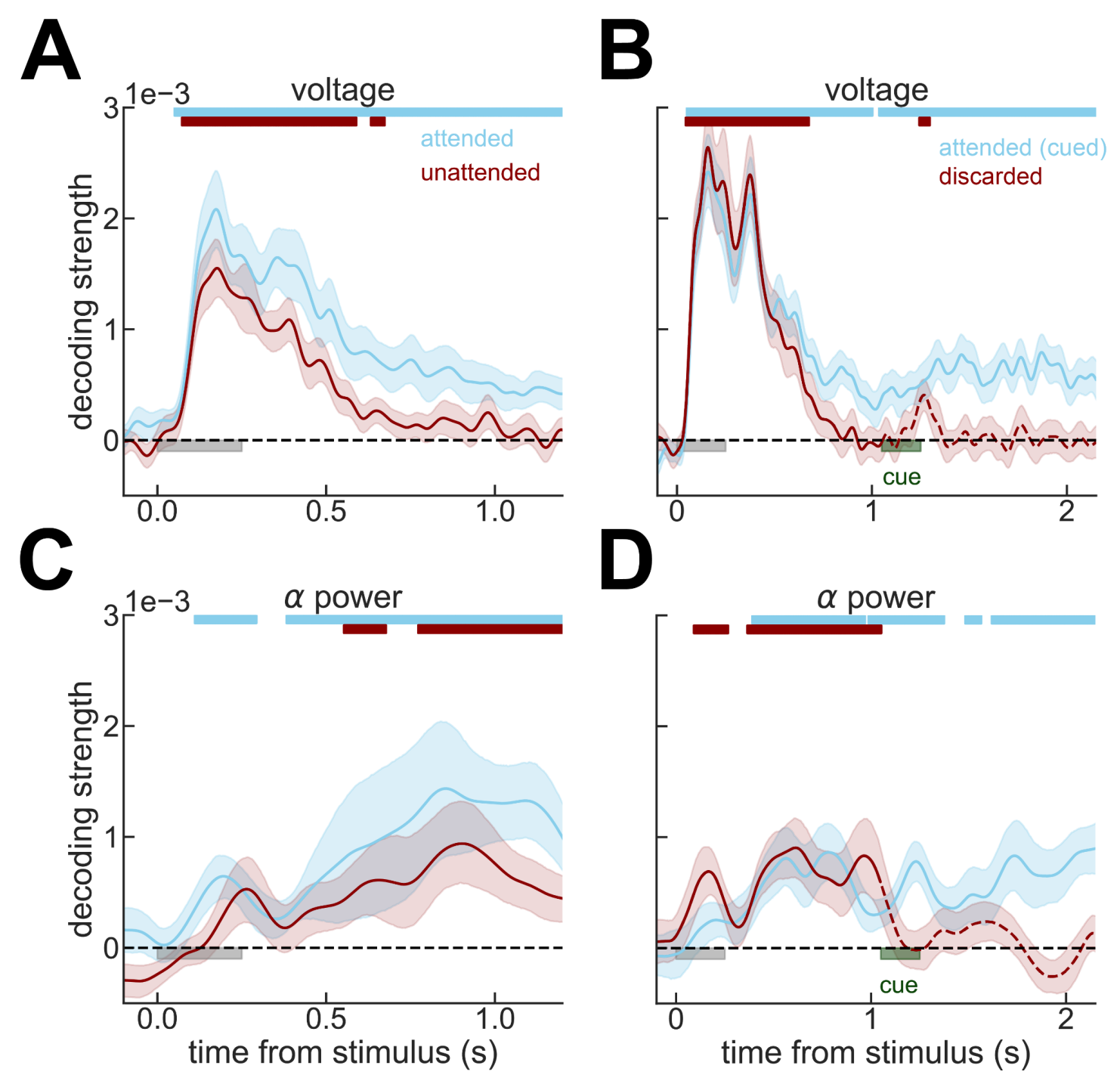

Figure 1. Decoding from alpha power reveals an underlying, active working memory code. A), Strength of stimulus decoding from raw voltage traces as in [7]. As in the original study, unattended or B) discarded memories cannot be decoded from raw voltage traces. C, D) Same as A) and B), but decoding from alpha power (Methods), which reveals a sustained representation of the unattended stimulus. In 1) and C), we analyse data from experiment 2 [7] while in B) and D), data from experiment 1 [7]. Light gray bars mark stimulus presentation periods. Notice that pinging stimulus presentation is not shown in this figure. Dashed lines mark the periods in which memories are irrelevant for upcoming behavior, following an instruction cue (dark green). All error bars are bootstrapped SEM and color bars on the top mark the periods where bootstrapped 95\% C.I. was above zero. 

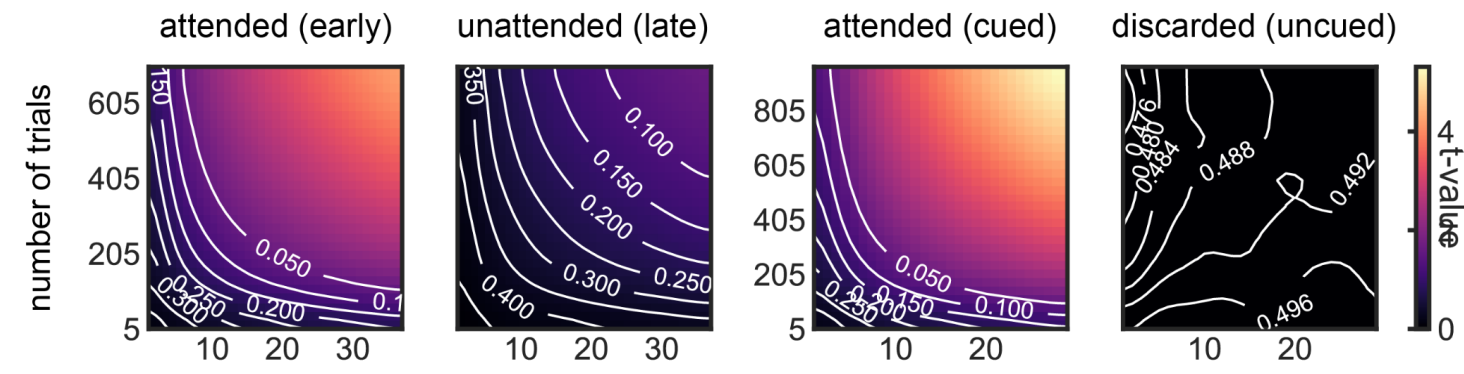

number of sessions

Figure 2. Voltage decoding as a function of trial and session numbers [61]. Attended items could be robustly decodable from voltage but not the unattended and discarded items. Note however the qualitative difference between unattended and discarded, especially how t-values along the diagonal increase clearly with sample size for unattended memories but not for discarded memories. Subsampling of sessions and trials was done by randomly subsampling $(n=5000)$ without repetition from the full dataset (Methods). In white, contour lines for different $p$-value levels (two-sided t-test). 


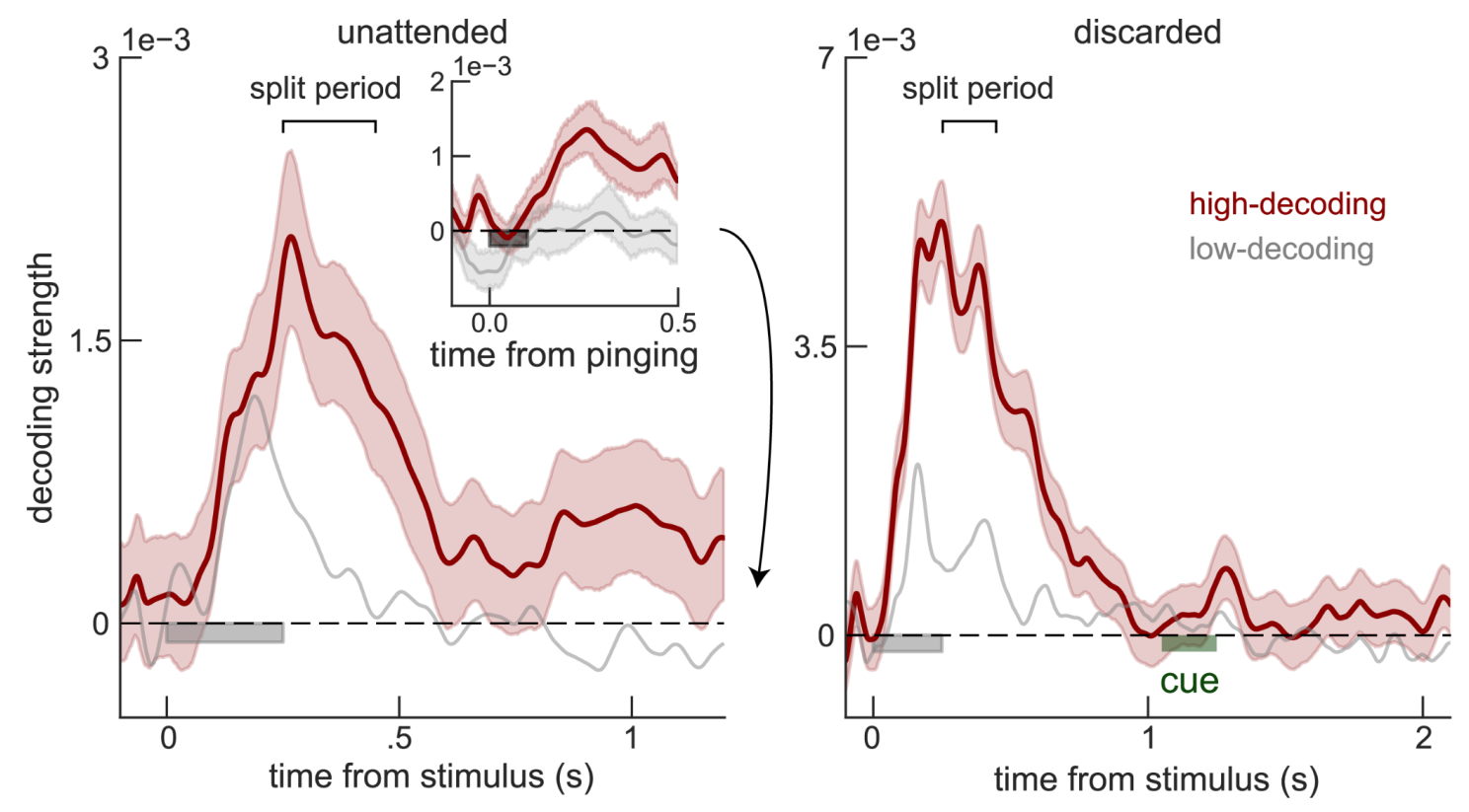

Figure 3. Sessions with high early-delay (split period, Methods) voltage decoding have a sustained code for unattended memories (left, red), but not for discarded memories (right). Replotting the reactivation period (inset), separately for high and low early-delay decoding sessions shows that "reactivations" only occur for sessions with a sustained code (left, red). Note that at time 0 the decoding strength is not actually zero (inset vs arrow). This is an artifact of baselining during the delay period (see also S3 Fig.). Error-bars are 90\% C.I., except for the inset where they are sem. Time-course and data are similar to Fig. 1A, B. 

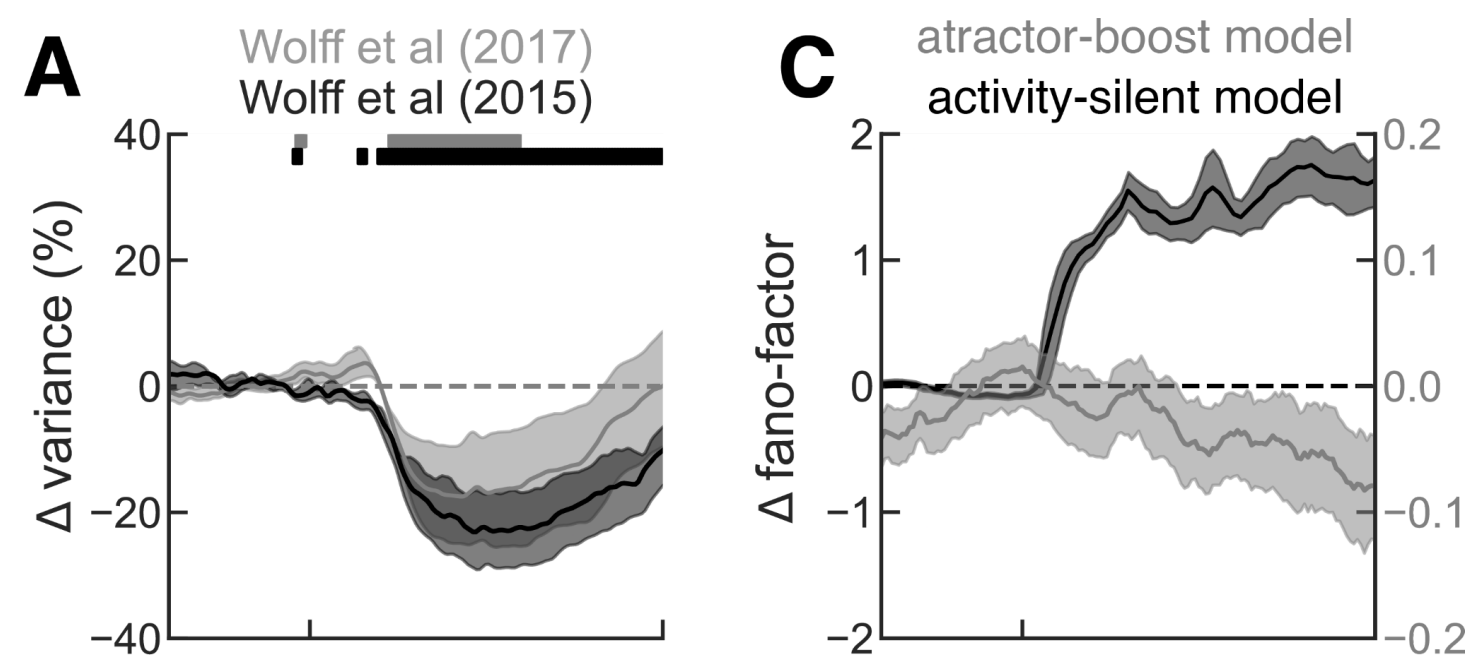

B variability median-split by
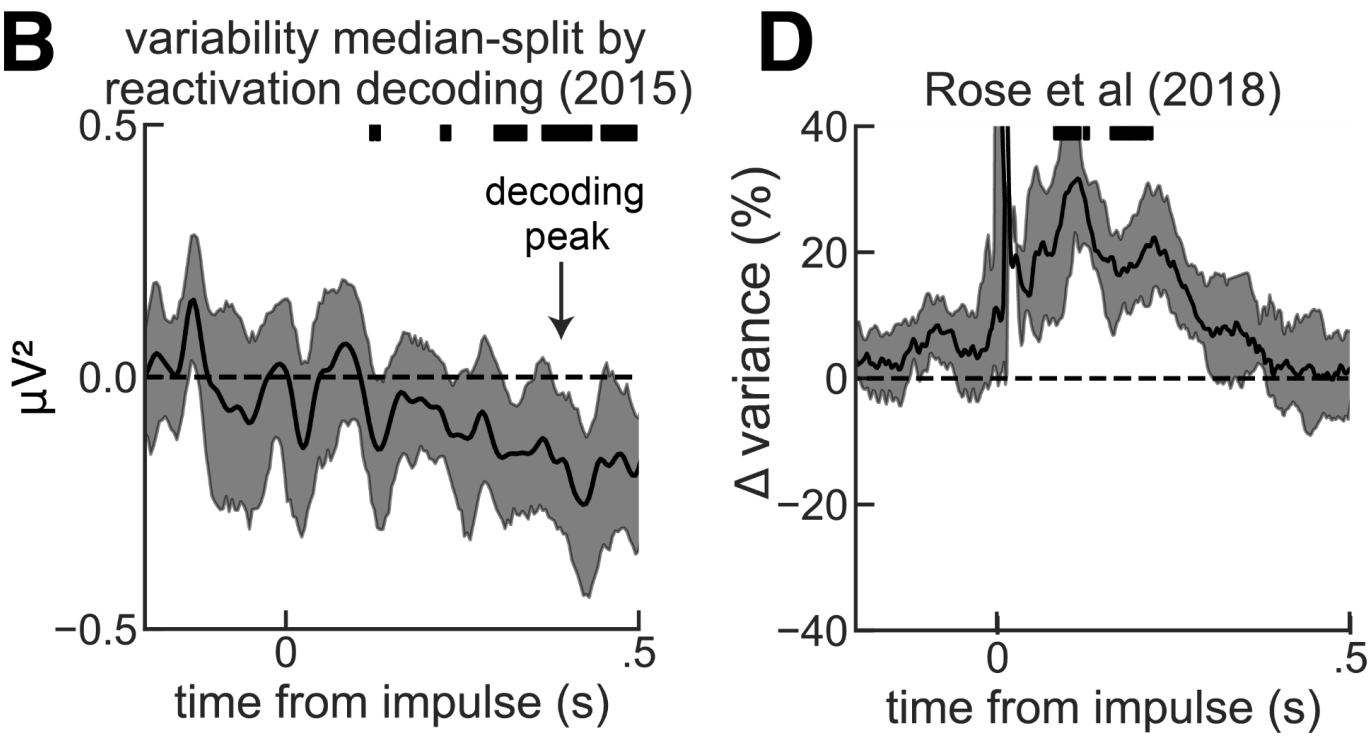

Figure 4. Impulse-induced, across-trial variability change in human EEG and computational models. A), percentage of variability change, relative to $.2 \mathrm{~s}$ before the impulse, computed when the impulse was a visual stimulus in Wolff et al (2017) in gray and Wolff et al (2015) in black. B), difference in variances computed across trials with low vs high stimulus decoding (computed at the time of maximal decodability, black arrow) in Wolff et al (2015). Trials with strong memory decoding showed significantly lower across-trial variance than trials with weak or absent memory decoding. We did not find a correlation in Wolff et al (2017) - data not shown - possibly because of a weaker pinging stimulus, which may have contributed to weaker increase in EEG decodability, not visible without baselining the data during the delay period (see S3 Fig.). C), simulations of the activity-silent working memory model with short-term plasticity (dark) predict an increase of across-trial variability (fano-factor) following reactivations induced by a non-specific drive. Simulations of the bump-attractor model without short-term plasticity (light) 
predict a decrease in the fano-factor following a non-specific drive. See also Supplementary Fig 3. D), same as A, but when the impulse was a single-pulse TMS. Solid bars mark where the change in variability was significant (two-sided t-test, $\mathrm{p}<0.005$ ) and error-bars are bootstrapped $95 \%$ C.I. of the mean. 


\section{Supplementary Figures}
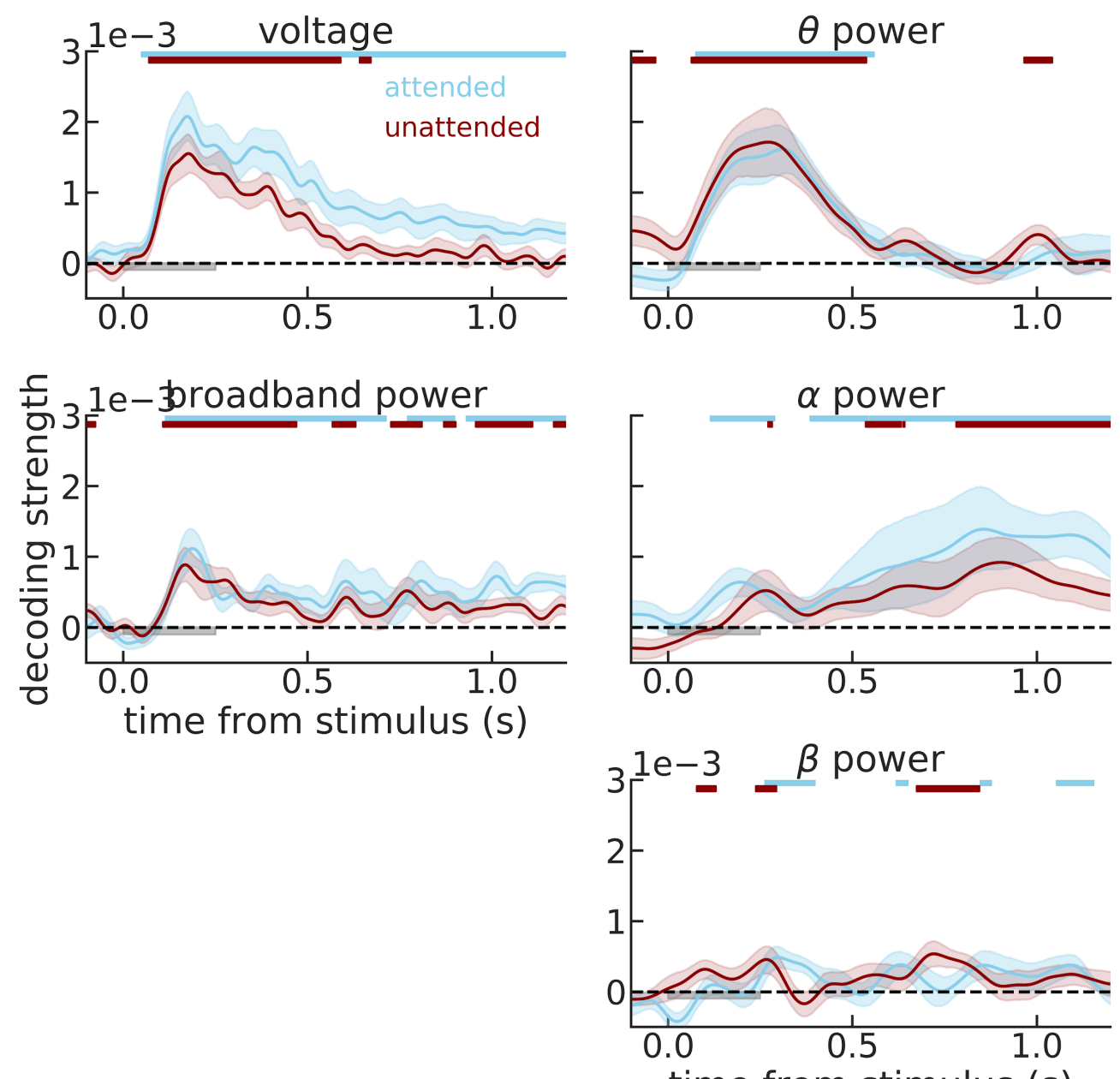

time from stimulus (s)

Supplementary Figure 1. A sustained alpha-power code tracks behaviorally relevant orientation memories. 

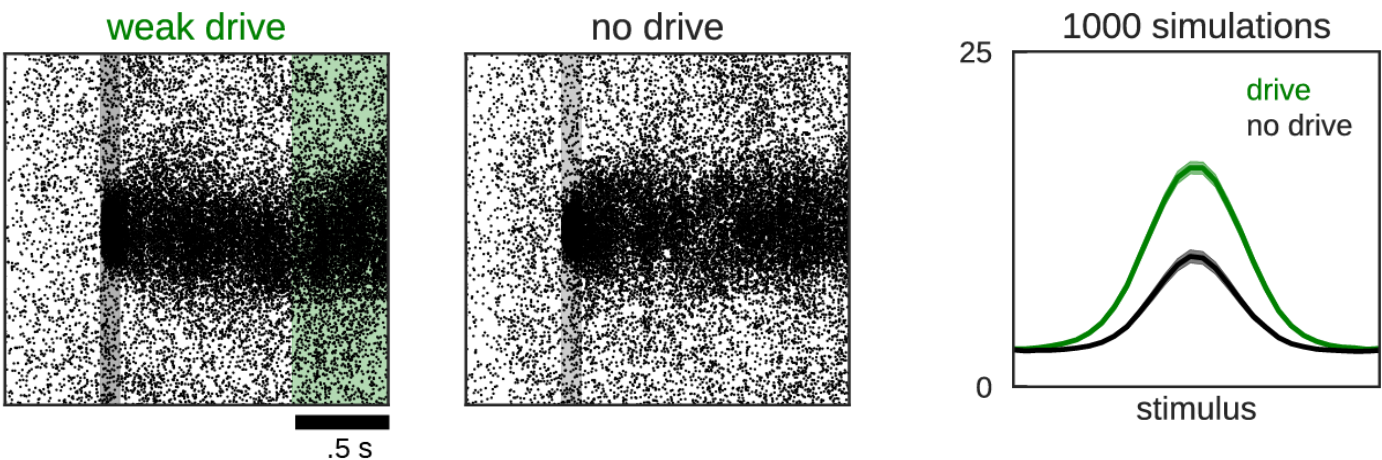

Supplementary Figure 2. In an attractor-boost network model, a non-specific stimulus increases tuning without reactivation. Two example stimulations of a bump-attractor with (weak drive) and without (no drive) a non-specific drive at the end of the trial. Importantly, we did not include short-term plasticity in either simulation, thus reactivations are not possible. Right, tuning during the last $.5 \mathrm{~s}$ is higher for the trials in which a non-specific drive was delivered (green), compared to when no drive was delivered (black). Similar models have been put forward in previous publications [29] (see also [62]). 


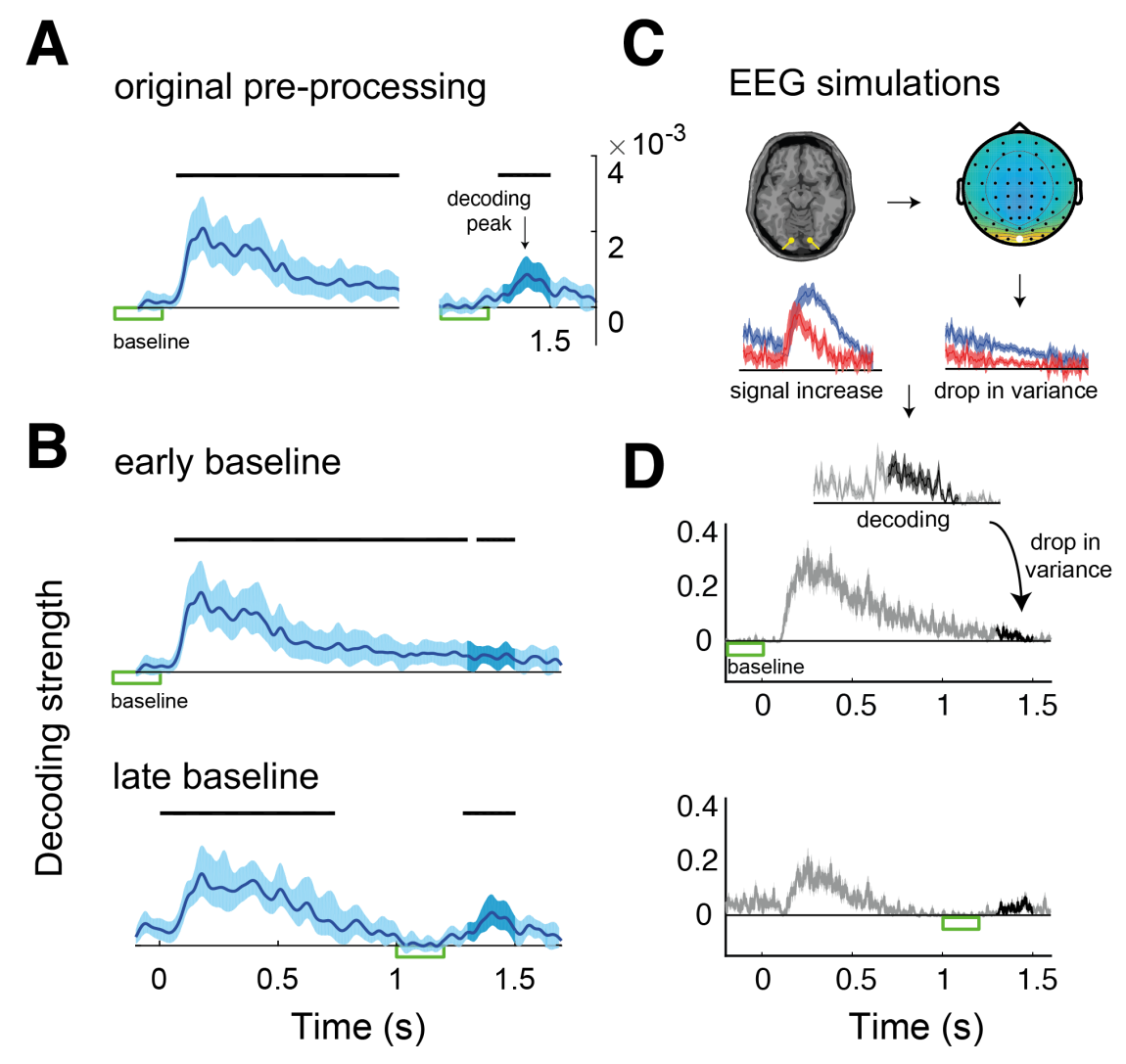

Supplementary Figure 3. Effect of EEG baselining on stimulus decoding analyses. A, original data from Wolff et al. (2017) was baselined twice. B, For our analyses, we de-baselined the second baselining of each trial so we could get continuity in EEG voltage traces through the whole trial (early baseline). Importantly, the exact time of the baseline affects the strength of 'reactivations'. Note that data without baselining (not shown) or with early baselining does not show any visible increase in EEG decoding. C, Top, diagram outlining the computer simulation that generated the EEG synthetic data, as if two current dipoles were placed within the visual cortex (yellow) to recreate artificial trials. Two different sets of trials $(n=200$, blue and red), corresponding to two different stimuli (e.g. $0^{\circ}, 45^{\circ}$ ) were generated (Methods). We simulated two event-related potentials (electrode 'Oz'; white) followed by a drop in across-trial variability. Bottom, illustration of how a similar signal-to-noise ratio increase (shown in 'decoding' for the drop in variance case) can result from a drop in variance or actual reactivation (signal increase). D, Through simulations we show that the baselining procedure introduces spurious reactivations in data without any true reactivation signal. By applying the decoding methods of Wolff et al. [7] we observe that (as in the data), spurious reactivations are barely visible with a distant baseline (early baseline), but are amplified for more proximal baselines (late 
baseline). These analyses illustrate that baselining is problematic and should be avoided, as it has been previously pointed out [28]. Darker lines mark the impulse period in the data and drop in variance in the simulations. 


\section{Author contributions}

$\mathrm{JB}, \mathrm{DL}$ and $\mathrm{AC}$ designed the study, JB and $\mathrm{DL}$ analysed the data. JB, AC developed the network simulations and DL developed the EEG simulations. JB wrote the first manuscript draft. JB and AC edited and reviewed the manuscript.

\section{Competing interests}

The authors declare no competing interests.

\section{Data availability}

All the code used for the analyses and simulations are available at https://github.com/comptelab/reactivations. The data and data decoder scripts are available from the original publications.

\section{Acknowledgments}

This work was funded by the Spanish Ministry of Science, Innovation and Universities and European Regional Development Fund (Refs: BFU2015-65315-R and RTI2018-094190-B-I00); by the Institute Carlos III, Spain (grant PIE 16/00014); by the Cellex Foundation; by the Generalitat de Catalunya (AGAUR 2014SGR1265, 2017SGR01565); and by the CERCA Programme/Generalitat de Catalunya. JB was supported by the Spanish Ministry of Economy and Competitiveness (FPI program) by the Bial (Ref: 356/18) and the Fyssen foundation. This work was developed at the building Centro Esther Koplowitz, Barcelona. We thank Nathan Rose for sharing part of Rose et al. 2016 dataset privately and the authors of Wolff et al. $(2017,2015)$ for sharing the full dataset publicly. We thank Michael Wolff and Mark Stokes for constructive discussions.

\section{Bibliography}

1. Mongillo G, Barak O, Tsodyks M. Synaptic theory of working memory. Science. 2008;319: 1543-1546. doi:10.1126/science.1150769 
2. Masse NY, Yang GR, Song HF, Wang X-J, Freedman DJ. Circuit mechanisms for the maintenance and manipulation of information in working memory. Nat Neurosci. 2019;22: 1159-1167. doi:10.1038/s41593-019-0414-3

3. Pals M, Stewart TC, Akyurek EG, Borst JP. A functional spiking-neuron model of activity-silent working memory in humans based on calcium-mediated short-term synaptic plasticity. BioRxiv. 2019. doi:10.1101/823559

4. Manohar SG, Zokaei N, Fallon SJ, Vogels TP, Husain M. Neural mechanisms of attending to items in working memory. Neurosci Biobehav Rev. 2019;101: 1-12. doi:10.1016/j.neubiorev.2019.03.017

5. Barbosa J, Stein H, Martinez RL, Galan-Gadea A, Li S, Dalmau J, et al. Interplay between persistent activity and activity-silent dynamics in the prefrontal cortex underlies serial biases in working memory. Nat Neurosci. 2020;23: 1016-1024. doi:10.1038/s41593-020-0644-4

6. Rose NS, LaRocque JJ, Riggall AC, Gosseries O, Starrett MJ, Meyering EE, et al. Reactivation of latent working memories with transcranial magnetic stimulation. Science. 2016;354: 1136-1139. doi:10.1126/science.aah7011

7. Wolff MJ, Jochim J, Akyürek EG, Stokes MG. Dynamic hidden states underlying working-memory-guided behavior. Nat Neurosci. 2017;20: 864-871. doi:10.1038/nn.4546

8. Masse NY, Rosen MC, Freedman DJ. Reevaluating the Role of Persistent Neural Activity in Short-Term Memory. Trends Cogn Sci (Regul Ed). 2020;24: 242-258. doi:10.1016/j.tics.2019.12.014

9. Beukers AO, Buschman TJ, Cohen JD, Norman KA. Is activity silent working memory simply episodic memory? Trends Cogn Sci (Regul Ed). 2021;25: 284-293. doi:10.1016/j.tics.2021.01.003

10. Oberauer K. Working Memory and Attention - A Conceptual Analysis and Review. J Cogn. 2019;2: 36. doi:10.5334/joc.58

11. Scimeca JM, Kiyonaga A, D'Esposito M. Reaffirming the sensory recruitment account of working memory. Trends Cogn Sci (Regul Ed). 2018;22: 190-192. doi:10.1016/j.tics.2017.12.007

12. Sreenivasan KK, D'Esposito M. The what, where and how of delay activity. Nat Rev Neurosci. 2019;20: 466-481. doi:10.1038/s41583-019-0176-7

13. Mallett R, Lewis-Peacock JA. Behavioral decoding of working memory items inside and outside the focus of attention. Ann N Y Acad Sci. 2018;1424: 256-267. doi:10.1111/nyas. 13647

14. Rademaker RL, Serences JT. Pinging the brain to reveal hidden memories. Nat Neurosci. 2017;20: 767-769. doi:10.1038/nn.4560

15. Orhan AE, Ma WJ. A diverse range of factors affect the nature of neural representations underlying short-term memory. Nat Neurosci. 2019;22: 275-283. doi:10.1038/s41593-018-0314-y 
16. Bliss DP, D'Esposito M. Synaptic augmentation in a cortical circuit model reproduces serial dependence in visual working memory. PLoS ONE. 2017;12: e0188927. doi:10.1371/journal.pone.0188927

17. Carter E, Wang X-J. Cannabinoid-mediated disinhibition and working memory: dynamical interplay of multiple feedback mechanisms in a continuous attractor model of prefrontal cortex. Cereb Cortex. 2007;17 Suppl 1: i16-26. doi:10.1093/cercor/bhm103

18. Wolff MJ, Ding J, Myers NE, Stokes MG. Revealing hidden states in visual working memory using electroencephalography. Front Syst Neurosci. 2015;9: 123. doi:10.3389/fnsys.2015.00123

19. LaRocque JJ, Lewis-Peacock JA, Drysdale AT, Oberauer K, Postle BR. Decoding attended information in short-term memory: an EEG study. J Cogn Neurosci. 2013;25: 127-142. doi:10.1162/jocn_a_00305

20. Sprague TC, Ester EF, Serences JT. Restoring latent visual working memory representations in human cortex. Neuron. 2016;91: 694-707. doi:10.1016/j.neuron.2016.07.006

21. Stokes MG, Kusunoki M, Sigala N, Nili H, Gaffan D, Duncan J. Dynamic coding for cognitive control in prefrontal cortex. Neuron. 2013;78: 364-375. doi:10.1016/j.neuron.2013.01.039

22. Watanabe K, Funahashi S. Neural mechanisms of dual-task interference and cognitive capacity limitation in the prefrontal cortex. Nat Neurosci. 2014;17: 601-611. doi:10.1038/nn.3667

23. Foster JJ, Sutterer DW, Serences JT, Vogel EK, Awh E. The topography of alpha-band activity tracks the content of spatial working memory. J Neurophysiol. 2016;115: 168-177. doi:10.1152/jn.00860.2015

24. Barbosa J. Working memories are maintained in a stable code. J Neurosci. 2017;37: 8309-8311. doi:10.1523/JNEUROSCI.1547-17.2017

25. Bae G-Y, Luck SJ. Dissociable Decoding of Spatial Attention and Working Memory from EEG Oscillations and Sustained Potentials. J Neurosci. 2018;38: 409-422. doi:10.1523/JNEUROSCI.2860-17.2017

26. Hakim N, Adam KCS, Gunseli E, Awh E, Vogel EK. Dissecting the Neural Focus of Attention Reveals Distinct Processes for Spatial Attention and Object-Based Storage in Visual Working Memory. Psychol Sci. 2019;30: 526-540. doi:10.1177/0956797619830384

27. Wolff MJ, Akyurek EG, Stokes MG. What is the functional role of delay-related alpha oscillations during working memory? 2021. doi:10.31234/osf.io/z6y5b

28. van Driel J, Olivers CNL, Fahrenfort JJ. High-pass filtering artifacts in multivariate classification of neural time series data. J Neurosci Methods. 2021;352: 109080. doi:10.1016/j.jneumeth.2021.109080

29. Schneegans S, Bays PM. Restoration of fMRI Decodability Does Not Imply Latent Working Memory States. J Cogn Neurosci. 2017;29: 1977-1994. 
doi:10.1162/jocn_a_01180

30. Christophel TB, lamshchinina P, Yan C, Allefeld C, Haynes J-D. Cortical specialization for attended versus unattended working memory. Nat Neurosci. 2018;21: 494-496. doi:10.1038/s41593-018-0094-4

31. lamshchinina P, Christophel TB, Gayet S, Rademaker RL. Essential considerations for exploring visual working memory storage in the human brain. Vis cogn. 2021; 1-12. doi:10.1080/13506285.2021.1915902

32. Wan Q, Cai Y, Samaha J, Postle BR. Tracking stimulus representation across a 2-back visual working memory task. R Soc Open Sci. 2020;7: 190228. doi: $10.1098 /$ rsos. 190228

33. van Loon AM, Olmos-Solis K, Fahrenfort JJ, Olivers CN. Current and future goals are represented in opposite patterns in object-selective cortex. elife. 2018;7. doi:10.7554/eLife.38677

34. Yu Q, Teng C, Postle BR. Different states of priority recruit different neural representations in visual working memory. PLoS Biol. 2020;18: e3000769. doi:10.1371/journal.pbio.3000769

35. Panichello MF, Buschman TJ. Selective control of working memory in prefrontal, parietal, and visual cortex. BioRxiv. 2020. doi:10.1101/2020.04.07.030718

36. Spaak E, Watanabe K, Funahashi S, Stokes MG. Stable and dynamic coding for working memory in primate prefrontal cortex. J Neurosci. 2017;37: 6503-6516. doi:10.1523/JNEUROSCI.3364-16.2017

37. Libby A, Buschman TJ. Rotational dynamics reduce interference between sensory and memory representations. Nat Neurosci. 2021;24: 715-726. doi:10.1038/s41593-021-00821-9

38. Churchland MM, Yu BM, Cunningham JP, Sugrue LP, Cohen MR, Corrado GS, et al. Stimulus onset quenches neural variability: a widespread cortical phenomenon. Nat Neurosci. 2010;13: 369-378. doi:10.1038/nn.2501

39. Barbosa J, Stein H, Martinez R, Galan A, Adam K, Li S, et al. Interplay between persistent activity and activity-silent dynamics in prefrontal cortex during working memory. BioRxiv. 2019. doi:10.1101/763938

40. Seeholzer A, Deger M, Gerstner W. Stability of working memory in continuous attractor networks under the control of short-term plasticity. PLoS Comput Biol. 2019;15: e1006928. doi:10.1371/journal.pcbi.1006928

41. Kilpatrick ZP. Synaptic mechanisms of interference in working memory. Sci Rep. 2018;8: 7879. doi:10.1038/s41598-018-25958-9

42. Fulvio JM, Postle BR. Cognitive control, not time, determines the status of items in working memory. J Cogn. 2020;3: 8. doi:10.5334/joc.98

43. de Azevedo Neto RM, Bartels A. Disrupting short-term memory in premotor but not visual cortex affects serial dependence in visuomotor integration. 
BioRxiv. 2021. doi:10.1101/2021.02.18.431802

44. Zokaei N, Manohar S, Husain M, Feredoes E. Causal evidence for a privileged working memory state in early visual cortex. J Neurosci. 2014;34: 158-162. doi:10.1523/JNEUROSCI.2899-13.2014

45. Wolff MJ, Jochim J, Akyürek EG, Buschman TJ, Stokes MG. Drifting codes within a stable coding scheme for working memory. PLoS Biol. 2020;18: e3000625. doi:10.1371/journal.pbio.3000625

46. Barbosa J. Neural network mechanisms of working memory interference. Doctoral dissertation, University of Barcelona. 2019.

47. Stokes MG, Muhle-Karbe PS, Myers NE. Theoretical distinction between functional states in working memory and their corresponding neural states. 2020. doi:10.31234/osf.io/n37su

48. Wimmer K, Nykamp DQ, Constantinidis C, Compte A. Bump attractor dynamics in prefrontal cortex explains behavioral precision in spatial working memory. Nat Neurosci. 2014;17: 431-439. doi:10.1038/nn.3645

49. Panichello MF, Buschman TJ. Shared mechanisms underlie the control of working memory and attention. Nature. 2021;592: 601-605. doi:10.1038/s41586-021-03390-w

50. Zucker RS, Regehr WG. Short-term synaptic plasticity. Annu Rev Physiol. 2002;64: 355-405. doi:10.1146/annurev.physiol.64.092501.114547

51. Zylberberg J, Strowbridge BW. Mechanisms of persistent activity in cortical circuits: possible neural substrates for working memory. Annu Rev Neurosci. 2017;40: 603-627. doi:10.1146/annurev-neuro-070815-014006

52. Itskov V, Hansel D, Tsodyks M. Short-Term Facilitation may Stabilize Parametric Working Memory Trace. Front Comput Neurosci. 2011;5: 40. doi:10.3389/fncom.2011.00040

53. Tegnér J, Compte A, Wang X-J. The dynamical stability of reverberatory neural circuits. Biol Cybern. 2002;87: 471-481. doi:10.1007/s00422-002-0363-9

54. Stein H, Barbosa J, Rosa-Justicia M, Prades L, Morató A, Galan-Gadea A, et al. Reduced serial dependence suggests deficits in synaptic potentiation in anti-NMDAR encephalitis and schizophrenia. Nat Commun. 2020;11: 4250. doi:10.1038/s41467-020-18033-3

55. Stokes MG. "Activity-silent" working memory in prefrontal cortex: a dynamic coding framework. Trends Cogn Sci (Regul Ed). 2015;19: 394-405. doi:10.1016/j.tics.2015.05.004

56. Bae G-Y, Luck SJ. Reactivation of previous experiences in a working memory task. Psychol Sci. 2019;30: 587-595. doi:10.1177/0956797619830398

57. Oostenveld R, Praamstra P. The five percent electrode system for high-resolution EEG and ERP measurements. Clin Neurophysiol. 2001;112: 713-719. doi:10.1016/S1388-2457(00)00527-7 
58. Tuckell HC. Introduction to Theoretical Neurobiology: Volume 2, Nonlinear and Stochastic Theories. Cambridge University Press 1988, editor. 1988.

59. Compte A, Brunel N, Goldman-Rakic PS, Wang XJ. Synaptic mechanisms and network dynamics underlying spatial working memory in a cortical network model. Cereb Cortex. 2000;10: 910-923. doi:10.1093/cercor/10.9.910

60. Goodman D, Brette R. Brian: a simulator for spiking neural networks in python. Front Neuroinformatics. 2008;2: 5. doi:10.3389/neuro.11.005.2008

61. Hajonides JE, Nobre AC, van Ede F, Stokes MG. Decoding visual colour from scalp electroencephalography measurements. Neuroimage. 2021;237: 118030. doi:10.1016/j.neuroimage.2021.118030

62. Edin F, Klingberg T, Johansson P, McNab F, Tegnér J, Compte A. Mechanism for top-down control of working memory capacity. Proc Natl Acad Sci USA. 2009;106: 6802-6807. doi:10.1073/pnas.0901894106 